\title{
Secondary prophylaxis in rheumatic fever: is it time to change?
}

\author{
Andrea Taddio ${ }^{1,2^{*}}$, A Pirrone ${ }^{2}$, Serena Pastore ${ }^{1}$, Loredana Lepore ${ }^{2}$, Caterina Di Battista ${ }^{3}$, Gabriele Simonini ${ }^{4}$, \\ Luciana Breda ${ }^{3}$, Rolando Cimaz ${ }^{4}$ \\ From 71st Congress of the Italian Society of Pediatrics. Joint National Meeting SIP, SIMGePeD, Study Group \\ on Pediatric Ultrasound, SUP Study Group on Hypertension \\ Rome, Italy. 4-6 June 2015
}

\section{Background}

Carditis and rheumatic chronic heart disease are the most serious complications of Acute Rheumatic Fever. Nowadays prevention of recurrent episodes of group A $\beta$-hemolytic streptococcal pharyngitis is the most effective method to prevent the development of severe rheumatic heart disease. However the evidence of these guidelines are weak and result from studies conducted more than 50 years ago [1]. To detect rate of Carditis related to Acute Rheumatic Fever found at follow up and to find a relationship with clinical data at diagnosis and compliance to prophylaxis.

\section{Material and methods}

This is a multicentre retrospective study conducted among 117 pediatric patients admitted with diagnosis of rheumatic carditis. We analysed the presence of carditis at diagnosis and at follow up comparing it with the number of infection recurrences and with the level of compliance of the patients to antibiotic prophylaxis. Compliance to antibiotic prophylaxis was evaluated individually with a questionnaire. The association between recurrences and compliance to therapy and between recurrences of streptococcal infections and carditis at follow up was also analyzed. Data were analysed using Fisher exact test.

\section{Results}

We examined 117 pediatric patients with rheumatic fever carditis. The median age of the patients at diagnosis was 9 years (6-11 years). The median age at follow up was 15 years, and the data at follow up were taken at a median time of 6,8 years from diagnosis. The data show that carditis at follow up was associated with the presence of carditis at diagnosis $(\mathrm{p}<0.000)$ and not with the level of compliance to antibiotic prophylaxis $(\mathrm{p}=\mathrm{NS})$. Also there was no statistically significant association between recurrences of infections and good level of compliance to therapy $(\mathrm{p}=\mathrm{NS})$ and between number of recurrences and pesence of carditis at follow up $(\mathrm{p}=\mathrm{NS})$.

\section{Conclusions}

We observed that the risk to develop carditis at follow up in pediatric patients with Acute Rheumatic Fever is independent from the compliance to antibiotic prophylaxis and the number of infection episodes caused by group A $\beta$-hemolytic Streptococcus while it seems related to the presence of carditis at time of diagnosis.

\footnotetext{
Authors' details

${ }^{1}$ Institute for Maternal and Child Health - IRCCS "Burlo Garofolo" - Trieste, Italy. ${ }^{2}$ University of Trieste, Trieste, Italy. ${ }^{3}$ Department of Paediatrics, University of Chieti, Chieti, Italy. ${ }^{4}$ AOU Meyer, Florence, Italy.
}

Published: 30 September 2015

\section{Reference}

1. Manyemba J, Mayosi BM: Penicillin for secondary prevention of rheumatic fever. Cochrane Database Syst Rev 2002, , 3: CD002227.

doi:10.1186/1824-7288-41-S2-A71

Cite this article as: Taddio et al:: Secondary prophylaxis in rheumatic fever: is it time to change? Italian Journal of Pediatrics 2015 41(Suppl 2): A71.

\footnotetext{
* Correspondence: andrea.taddio@burlo.trieste.it

${ }^{1}$ Institute for Maternal and Child Health - IRCCS "Burlo Garofolo" - Trieste,

Italy

Full list of author information is available at the end of the article
} 